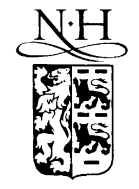

ELSEVIER

\title{
Growth, crystalline structure and magnetic properties of ultrathin alloy films $\mathrm{Co}_{x} \mathrm{Ni}_{1-x} / \mathrm{Cu}\left(\begin{array}{lll}1 & 0 & 0\end{array}\right)$
}

\author{
W.C. Lin, C.C. Kuo, C.L. Chiu, Minn-Tsong Lin * \\ Department of Physics, National Taiwan University, 1, Sec. 4, Roosevelt Road, 10617 Taipei, Taiwan
}

Received 1 August 2000; accepted for publication 5 February 2001

\begin{abstract}
Magnetic ultrathin alloy films $\mathrm{Co}_{x} \mathrm{Ni}_{1-x} / \mathrm{Cu}(100)$ were prepared to characterize the behavior of spin reorientation transition (SRT). With the double calibration by medium energy electron diffraction oscillation and Auger electron spectroscopy, the composition of the alloy films was controlled within an accuracy of $\pm 0.5 \%$, which allowed us to prepare alloy films in very low Co concentration. The critical thickness of SRT in alloy ultrathin films $\mathrm{Co}_{x} \mathrm{Ni}_{1-x} l$ $\mathrm{Cu}(100)$ was significantly delayed for $x$ less than $10 \%$, and for $x=10 \%$, alloy films, at least up to $20.6 \mathrm{ML}$, always revealed in-plane anisotropy. Besides the sensitive influence of composition on SRT behavior when $x \leqslant 10 \%$, the vertical interlayer distance of all the films did not show any significant difference. These results were consistent with prior theoretical calculation, in which the magnetic anisotropy was concluded to change sign abruptly while tuning a few average number of $3 \mathrm{~d}$ electron. (c) 2001 Published by Elsevier Science B.V.
\end{abstract}

Keywords: Magnetic films; Magnetic measurements; Single crystal surfaces; Alloys; Auger electron spectroscopy; Low energy electron diffraction (LEED)

\section{Introduction}

In magnetic ultrathin films, owing to the reduced coordination, the surface effect on magnetic behavior is more significant than in the bulk. For example, thin films within several monolayers may have their surface anisotropy more important than the volume term. Therefore if the two terms prefer different moment orientation, by changing the film thickness or the temperature, their competition may lead to spin reorientation transition (SRT).

\footnotetext{
${ }^{*}$ Corresponding author. Tel.: +886-2-33665173; fax: +886-223639984.

E-mail address: mtlin@phys.ntu.edu.tw (M.-T. Lin).
}

These temperature and thickness induced SRT have been observed in various systems such as $\mathrm{Fe} /$

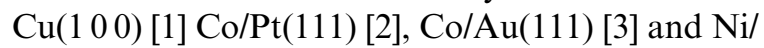

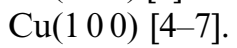

As a special case, $\mathrm{Ni} / \mathrm{Cu}\left(\begin{array}{lll}1 & 0 & 0\end{array}\right)$ films present very different SRT behaviors [4-7], in which the negative surface anisotropy results in the in-plane magnetization at low coverages (up to 7-8 ML), and the positive volume term brings the easy axis to be perpendicular with increasing thickness. This perpendicular magnetization exists until 30 $70 \mathrm{ML}$ in different experiments, then the easy axis switches to the in-plane direction. The origin of this stable perpendicular anisotropy in $\mathrm{Ni} /$ $\mathrm{Cu}\left(\begin{array}{lll}1 & 0 & 0\end{array}\right)$ has been understood as being caused by strain-induced anisotropy in the volume term. 
Because of the strain relaxation with increasing thickness, the anisotropy comes back to be inplane again [4-7]. In contrast to the $\mathrm{Ni} / \mathrm{Cu}\left(\begin{array}{lll}1 & 0 & 0\end{array}\right)$ system, $\mathrm{Co} / \mathrm{Cu}\left(\begin{array}{ll}0 & 0\end{array}\right)$ has a stable easy axis in the plane of the film $[8,9]$ due to its negative straininduced anisotropy $[10,11]$. Therefore, if Co and $\mathrm{Ni}$ were combined to form alloy films on $\mathrm{Cu}\left(\begin{array}{lll}1 & 0 & 0\end{array}\right)$, the magnetoelastic coefficient may change sign while increasing the Co concentration. Furthermore other magnetic properties such as magnetic moment and crystalline anisotropy may also change with the average number of $3 \mathrm{~d}$ electrons.

It has been predicted in many theoretical calculations that the crystalline and the straininduced anisotropy may change abruptly or smoothly with the average number of $3 \mathrm{~d}$ electrons [12-14]. This can be checked experimentally in magnetic alloy ultrathin films by varying the alloy composition, that means tuning the average number of $3 \mathrm{~d}$ electron. The composition-driven SRT has been observed to occur in $\mathrm{Fe}_{x} \mathrm{Co}_{1-x} / \mathrm{Cu}\left(\begin{array}{lll}1 & 0 & 0\end{array}\right)$ system without structural transition [15]. However, the previous experimental studies for the alloy films $\mathrm{Co}_{x} \mathrm{Ni}_{1-x} / \mathrm{Cu}\left(\begin{array}{lll}0 & 0\end{array}\right)$ always exhibited the inplane anisotropy without any SRT observed with $x$ ranging from $10 \%$ to $100 \%$ and film thickness up to $9 \mathrm{ML}$ [16]. So far, no further experiments have been performed for the $\mathrm{Co}_{x} \mathrm{Ni}_{1-x} / \mathrm{Cu}\left(\begin{array}{lll}1 & 0 & 0\end{array}\right)$ system, and the SRT behavior in the range of $x \leqslant 10 \%$ is still an interesting subject. Thus, ultrathin alloy films $\mathrm{Co}_{x} \mathrm{Ni}_{1-x} / \mathrm{Cu}(100)$ for $x \leqslant 10 \%$ were prepared in this experiment. The growth, crystalline structure and magnetic properties are studied to characterize the relationship between them and the alloy concentration.

\section{Experiment}

A polished single crystal $\mathrm{Cu}(100)$ with miscut $\leqslant 0.5^{\circ}$ was used as the substrate, which was prepared by cycles of $2 \mathrm{keV} \mathrm{Ar}$ ion sputtering followed by annealing to $800 \mathrm{~K}$ for $5 \mathrm{~min}$ to achieve a flat and clean surface. At room temperature ( $300 \mathrm{~K}), \mathrm{Co}$ and $\mathrm{Ni}$ were coevaporated onto the substrate using two evaporation guns (EFM-3, OMICRON). The base pressure of the UHV chamber was better than $5 \times 10^{-10}$ mbar and up to
$1 \times 10^{-9}$ mbar during the codeposition of $\mathrm{Co}$ and $\mathrm{Ni}$ onto $\mathrm{Cu}(100)$. The process of film growth was monitored by medium energy electron diffraction (MEED) such that information about the morphology can be observed. As long as the parameters of the evaporation guns were repeated, the MEED oscillation curve could be obtained with its peaks at almost the same timing as the previous MEED data. Since each peak of the MEED oscillation curve in the layer-by-layer growth means the completeness of each monolayer, the deposition rate can be determined from the timing of the peaks and thus the film thickness can be known. Besides, because it took at least several hundred seconds to deposit a thin film, the possible delay of opening or closing the shutters, which is less than 1 $\mathrm{s}$ is negligible. Therefore the reliability of the deposition rate helped to determine the film thickness precisely within $0.05 \mathrm{ML}$.

A precise determination of alloy composition is one of the key points in this work. Two independent ways were chosen to check the composition of the alloy ultrathin films. One is precisely controlling the deposition rate of $\mathrm{Co}$ and $\mathrm{Ni}$, which can be calibrated by MEED. Because of the definite repeat of the deposition rate by precisely controlling the experimental parameters of evaporation guns, it was possible to prepare samples in desired composition within an accuracy of $\pm 0.5 \%$ concentration value.

The other method is checking the concentration by Auger electron spectroscopy (AES). Although the AES peaks of $\mathrm{Co}, \mathrm{Ni}$ and $\mathrm{Cu}$ highly overlap, as shown in Fig. 1(a), the calibration of the composition can still be performed with an accuracy of $\pm 2 \%$ by quantitative AES analysis [17]. At first we prepared various $\mathrm{ML} \mathrm{Co} / \mathrm{Cu}(100)$ and $\mathrm{Ni} /$ $\mathrm{Cu}\left(\begin{array}{lll}1 & 0 & 0\end{array}\right)$ and took their AES signal as the reference bases. The only peak of $\mathrm{Cu}$, which does not overlap with those of $\mathrm{Co}$ and $\mathrm{Ni}$ is the primary peak at $920 \mathrm{eV}$ (Fig. 1(a)). From this peak, we can separate the AES signal of $\mathrm{Cu}$ from the mixing curves. For example, as depicted in Fig. 1(b), the Auger electron spectrum of pure bulk $\mathrm{Cu}$ was reduced to the same peak to peak height of the 920 $\mathrm{eV}$ peak as that of $7 \mathrm{ML} \mathrm{Ni} / \mathrm{Cu}(100)$. The reduced Auger electron spectrum was supposed to be the signal from the substrate $\mathrm{Cu}\left(\begin{array}{lll}1 & 0 & 0\end{array}\right)$ under $7 \mathrm{ML}$ Ni. 


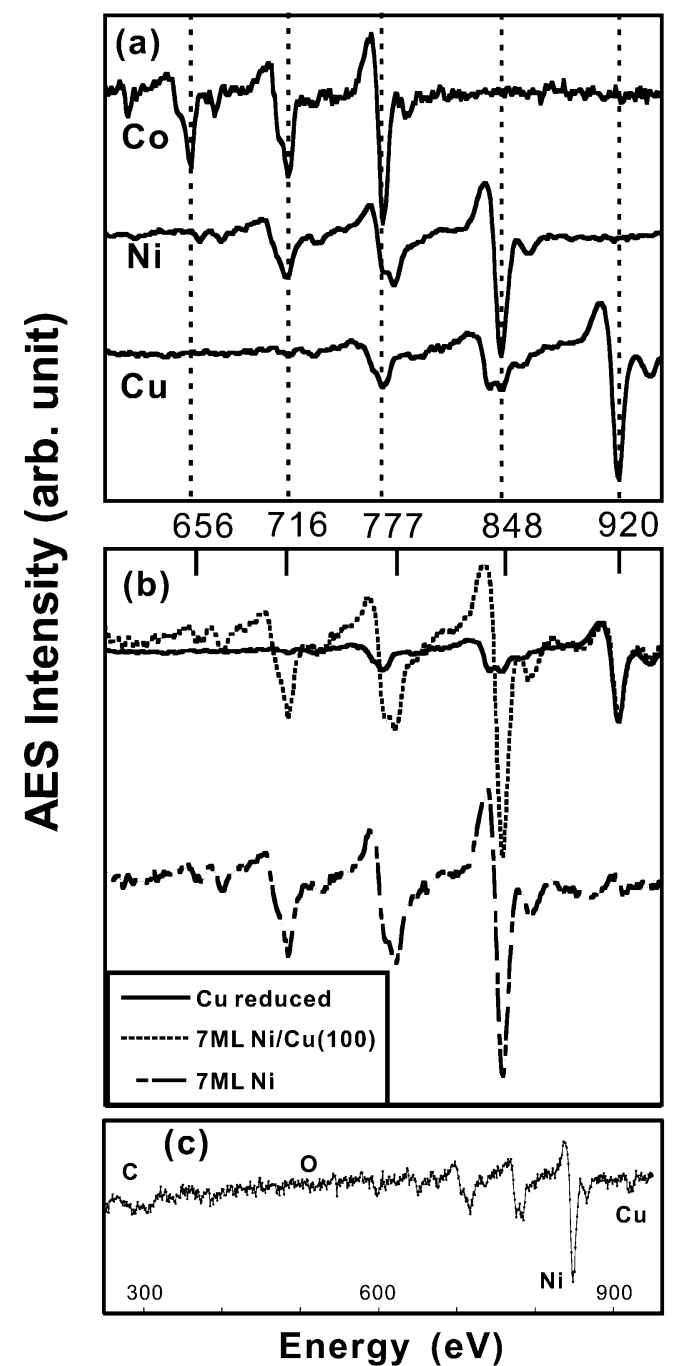

Fig. 1. (a) Auger electron spectra of bulk $\mathrm{Co}, \mathrm{Ni}$, and $\mathrm{Cu}$ measured with $3 \mathrm{keV}$ incident electron energy. It indicates that the characteristic peaks of $\mathrm{Co}, \mathrm{Ni}$, and $\mathrm{Cu}$ are highly overlaid. (b) By subtracting the AES signal of $\mathrm{Cu}$, we can get the pure Auger electron spectrum of 7 ML Ni. (c) Auger spectrum of 13 $\mathrm{ML} \mathrm{Ni} / \mathrm{Cu}(100)$ with its energy extending to the region of carbon and oxygen.

By subtracting the reduced Auger electron spectrum of $\mathrm{Cu}$ from that of $7 \mathrm{ML} \mathrm{Ni} / \mathrm{Cu}(100)$, we can get the AES curve of $7 \mathrm{ML} \mathrm{Ni}$, as shown in Fig. 1(b). In this way, the individual Auger electron spectra of various ML Co and Ni can be taken. Additionally, we can also get the Auger electron

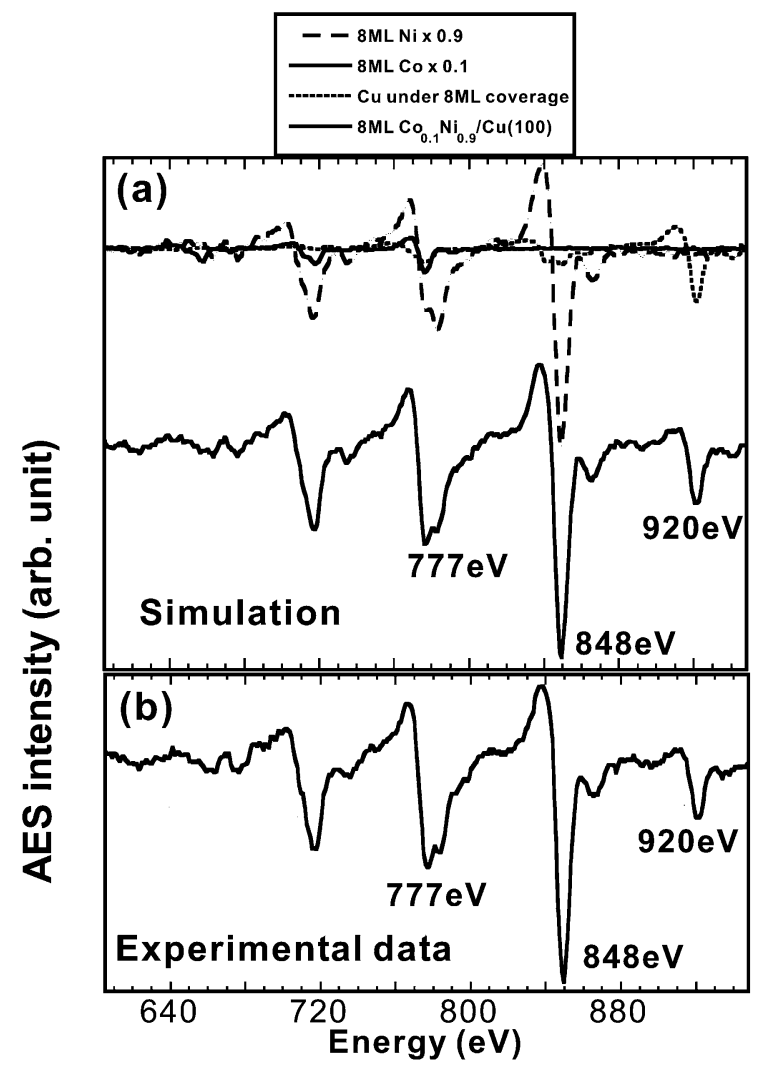

Fig. 2. (a) By linearly combining the signal of various elements, the AES curves of $8 \mathrm{ML} \mathrm{Co}_{0.1} \mathrm{Ni}_{0.9} / \mathrm{Cu}(100)$ can be simulated. (b) The experimental data agree well with the simulation both in the shape and the ratio of peaks.

spectra of the substrate $\mathrm{Cu}(100)$, which was under films of various thickness.

Finally, the Auger electron spectra of various alloy films $\mathrm{Co}_{x} \mathrm{Ni}_{1-x} / \mathrm{Cu}(100)$ was simulated. In Fig. 2(a), the AES peaks of $8 \mathrm{ML} \mathrm{Ni}$ and Co were reduced as $90 \%$ and $10 \%$ of the original ones, respectively, and then were combined to form the Auger electron spectrum of $8 \mathrm{ML} \mathrm{Co}_{0.1} \mathrm{Ni}_{0.9}$. Next, by combining the AES signal of $8 \mathrm{ML}$ films $\mathrm{Co}_{0.1} \mathrm{Ni}_{0.9}$ and that of the substrate $\mathrm{Cu}(100)$ under 8 ML coverage, Auger electron spectrum of $\mathrm{Co}_{0.1} \mathrm{Ni}_{0.9} / \mathrm{Cu}(100)$ can be simulated. The predicted AES ratio of $848 \mathrm{eV} / 777 \mathrm{eV}$ and $920 \mathrm{eV} / 848$ $\mathrm{eV}$ which gives information about the alloy composition and film thickness, respectively, are agreed well with our experimental data (Fig. 2(b)). In this method, it was unnecessary to fit any model 
parameters. What we needed were the AES spectra

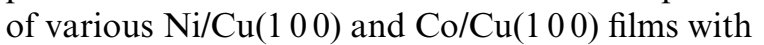
their thickness precisely determined by MEED. Then by the processes described as above, the simulation could be done. By the consistent results of MEED and AES, the compositions of alloy ultrathin films can be confirmed precisely.

A small Auger signal of carbon was observed and it was hard to observe oxygen in the films. Therefore the contamination of $\mathrm{CO}$ existed but was not serious. An example was shown in Fig. 1(c). It is the Auger spectrum of $13 \mathrm{ML} \mathrm{Ni} /$ $\mathrm{Cu}\left(\begin{array}{lll}0 & 0\end{array}\right)$ with its energy extending to the region of carbon and oxygen.

The growth of the alloy films was monitored by MEED which was performed with the electron energy of $2 \mathrm{keV}$. After the thin film deposition, the crystalline structure was detected by low energy electron diffraction (LEED) and $I / V$-LEED measurement at $200 \mathrm{~K}$. From the Bragg condition and the de Broglie relation, we have

$2 d \sin \theta=n \lambda=n \frac{h}{p}=n \frac{h}{\sqrt{2 m\left(E_{\mathrm{k}}-V\right)}}$

with $d$ the vertical interlayer distance, $E_{\mathrm{k}}$ the kinetic energy of the incident electron beam, $\theta$ the diffraction angle, and $V$ the potential cost for electrons to escape from the atoms. Eq. (1) can be rewritten as

$E_{\mathrm{k}}=n^{2} \frac{h^{2}}{8 m d^{2} \sin ^{2} \theta}+V$

For example, Fig. 3(a) is the $I / V$-LEED curve of bulk $\mathrm{Cu}$, which shows the $(00)$ beam intensity in different beam energy. If $E_{\mathrm{k}}$ and $n^{2}$ are taken as the $Y$ and $X$ axis, respectively, we can fit the peaks of $I / V$-LEED curve to Eq. (2), as shown in Fig. 3(b). Since $h, \theta$ and $m$ are known, $d$ can be got from the prefactor of $n^{2}$. This method has given the useful information on the average vertical interlayer distance in many other systems $[18,19]$.

The polar and longitudinal MOKE were performed in situ to characterize the magnetic properties. The applied field was aligned along the $\left[\begin{array}{lll}0 & 1 & 0\end{array}\right]$ direction for the in-plane measurement. In the MOKE measurement, a modulated $\mathrm{He}-\mathrm{Ne}$ laser beam was used as the light source such that

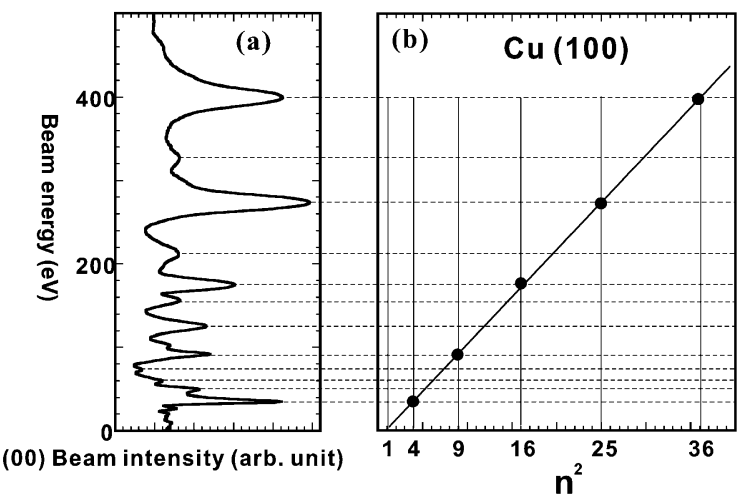

Fig. 3. (a) $I / V$-LEED curve of bulk $\mathrm{Cu}\left(\begin{array}{lll}1 & 0 & 0\end{array}\right)$ measured at 200 K. (b) The fitting of (b) to Eq. (2). The vertical interlayer distance can be got from the slope.

the periodic signal from magnetic ultrathin films can be taken out by lock-in technique with a high signal to noise ratio.

\section{Results and discussion}

In ultrathin films, it has been found in recent decades that the magnetic properties such as magnetic moment and anisotropy are very sensitive to the surface morphology and crystalline structure which may change with different growth condition $[1,18,20,21]$. Therefore in this experiment, the analysis of morphology and crystalline structure were made to realize that if they should be responsible for the change of the magnetic behavior with alloy concentration.

In Fig. 4, all the MEED intensity oscillations of the alloy samples grown at $300 \mathrm{~K}$ reveal nearly the same feature as that of pure $\mathrm{Ni} / \mathrm{Cu}\left(\begin{array}{lll}1 & 0 & 0\end{array}\right)$ [22]. The first four peaks of the MEED oscillation can be identified clearly, indicating that the layer-by-layer growth mode applies up to the fourth layer. The above result is quite consistent with the STM study in $\mathrm{Ni} / \mathrm{Cu}\left(\begin{array}{ll}1 & 0\end{array}\right)$ by Shen et al. [23]. $\mathrm{Co} / \mathrm{Cu}(100)$, as shown in Fig. 4, has a feature of bilayer growth mode in the first two ML and the layer-by-layer growth persists up to more than $8 \mathrm{ML}$ at room temperature [16]. However, this behavior seems to have no effect on our alloy films for $x$ up to $10 \%$. It 


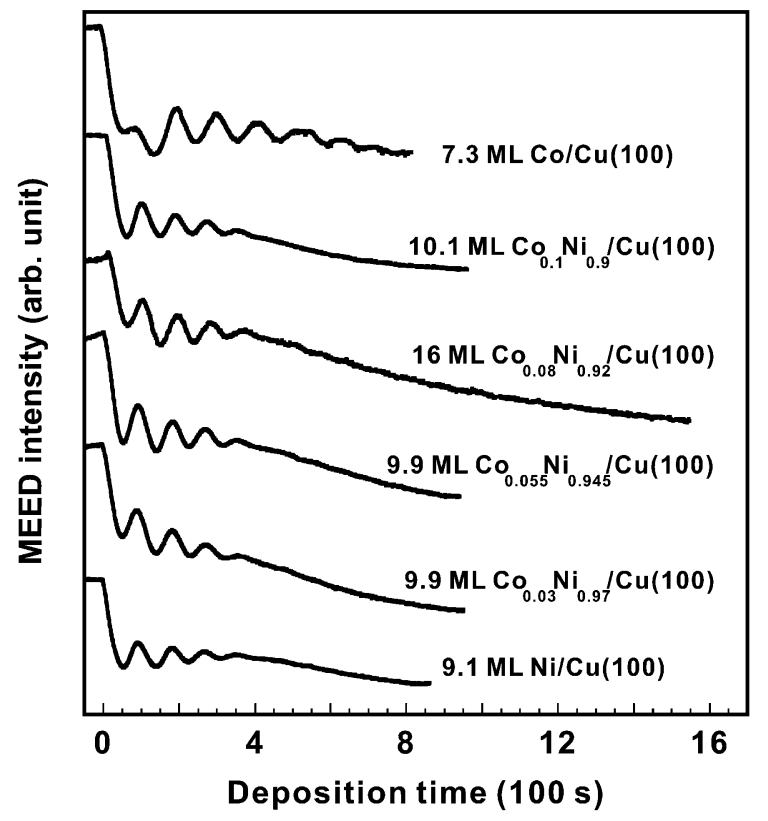

Fig. 4. MEED oscillation of ultrathin films with different Co concentration grown at $300 \mathrm{~K}$.

is not surprising that in such low Co concentration, Ni dominates the growth behavior.

Unlike the sharp circular LEED spots in $\mathrm{Cu}(100)$ (Fig. 5(a)), all the ultrathin films such as $\mathrm{Ni} / \mathrm{Cu}(100)$ and $\mathrm{Co}_{0.08} \mathrm{Ni}_{0.92} / \mathrm{Cu}(100)$ show the same crossing streaks in the LEED spots (Fig. 5(b) and (c)). After annealing to $350 \mathrm{~K}$ for $10 \mathrm{~min}$, these crossing streaks disappeared as depicted in Fig. 5(d). This feature is attributed to the rectangular 3D islands which have been observed in the STM study of $\mathrm{Ni} / \mathrm{Cu}\left(\begin{array}{lll}1 & 0 & 0\end{array}\right)[23,24]$. The 3D platelike islands form the additional periodic structure in the [0 $\left.\begin{array}{lll}0 & 1\end{array}\right]$ and [ $\left[\begin{array}{lll}0 & 1\end{array}\right]$ directions, resulting in the crossing streaks along these two directions. Since annealing at $350 \mathrm{~K}$ smoothed the films, which also has been realized in the STM study, the LEED patterns recover its circular spots as that of $\mathrm{Cu}(100)$. From the same feature of crossing streaks in the LEED patterns, all the alloy films are believed to have nearly the same structure ordering as $\mathrm{Ni} / \mathrm{Cu}(100)$.

Fig. 6(a) gives the LEED intensity curves of $(00)$ beam recorded at $200 \mathrm{~K}$ in different beam energy for various alloy films. The solid lines mark the
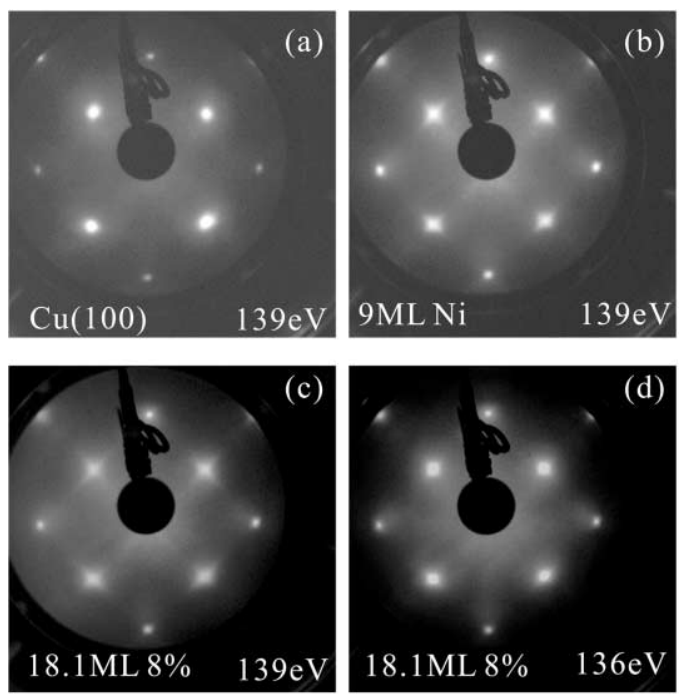

Fig. 5. LEED patterns of (a) $\mathrm{Cu}(100)$, (b) $9 \mathrm{ML} \mathrm{Ni/Cu(100)}$ and (c) $18.1 \mathrm{ML} \mathrm{Co}_{0.08} \mathrm{Ni}_{0.92} / \mathrm{Cu}(100)$, measured at $200 \mathrm{~K}$ with $E_{\mathrm{k}}=139 \mathrm{eV}$. Only (a) presents sharp circular spots. (b) and (c) show the apparent crossing streaks in the [ $\left[\begin{array}{lll}0 & 1 & 1\end{array}\right]$ and $\left[\begin{array}{lll}0 & 1 & 1\end{array}\right]$ directions denoting the edges of rectangular islands in the films. (d) After annealing to $350 \mathrm{~K}$ for $10 \mathrm{~min}$, the crossing streaks nearly disappear.

positions of Bragg condition, with which the vertical interlayer distance could be determined. There is no apparent difference in the positions of Bragg condition of various films. As shown in Fig. 6(b), the alloy films almost have the same vertical interlayer distance within the error of $\pm 0.02 \AA$. Unlike $\mathrm{Fe} / \mathrm{Cu}(100)$ which shows a phase transition from fcc to bcc structure [1], all the $\mathrm{Co}_{x} \mathrm{Ni}_{1-x} l$ $\mathrm{Cu}(100)$ films have the same structure within the error bars in different thickness. Comparing with the lattice constant of $\mathrm{Ni}$ bulk, the vertical interlayer distance is a little bit smaller due to the tensile strain of the film on $\mathrm{Cu}(100)$. It can also be found in the data of Fig. 6(b) that since all the ultrathin films keep the same deviation from the bulk in the vertical interlayer distance, the relaxation seems not to happen in the above alloy films. In the previous study of $\mathrm{Ni} / \mathrm{Cu}(100)$, the structure relaxation was observed when thickness is larger than 9-13 ML [5,25]. It is believable that in the alloy ultrathin films, the critical thickness of relaxation could be delayed by adding the Co concentration due to smaller mismatch of $\mathrm{Co}$ on $\mathrm{Cu}\left(\begin{array}{lll}1 & 0 & 0)\end{array}\right)$ than $\mathrm{Ni}$. 

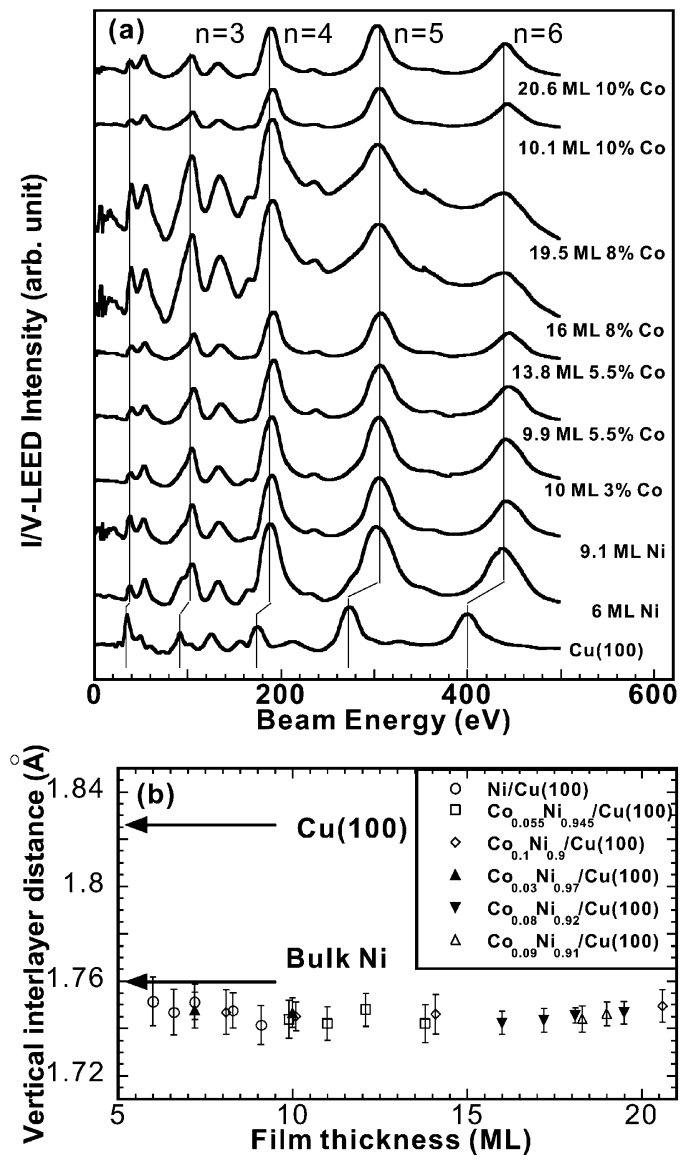

Fig. 6. (a) Intensity vs. electron energy of LEED ( 00$)$ beam for clean $\mathrm{Cu}(100)$ and various $\mathrm{ML} \mathrm{Co}_{x} \mathrm{Ni}_{1-x} /(100)$, measured at $200 \mathrm{~K}$. The solid lines denote the beam energy at which the Bragg conditions of the same index were reached. (b) The vertical interlayer distance of various alloy films, which were fitted from the above peaks.

It can be seen clearly in the magnetic hysteresis loops measured at $110 \mathrm{~K}$ by in situ MOKE (Fig. 7) that ultrathin films in different alloy composition do have a SRT at different critical thickness $d_{\mathrm{c}}$. As compared with $\mathrm{Ni} / \mathrm{Cu}\left(\begin{array}{lll}1 & 0 & 0\end{array}\right)$ films, the critical thickness of SRT was strongly delayed by only $3 \%$ and $5.5 \%$ Co in alloy films. This effect was stronger with the more Co concentration. Increasing the Co concentration also changed the shapes of hysteresis loops. The polar and longitudinal hysteresis loops of $\mathrm{Ni} / \mathrm{Cu}(100)$ films above $d_{\mathrm{c}}$ are more square than that for 3\% and 5.5\% Co. Similar results were also observed in other series of speci-

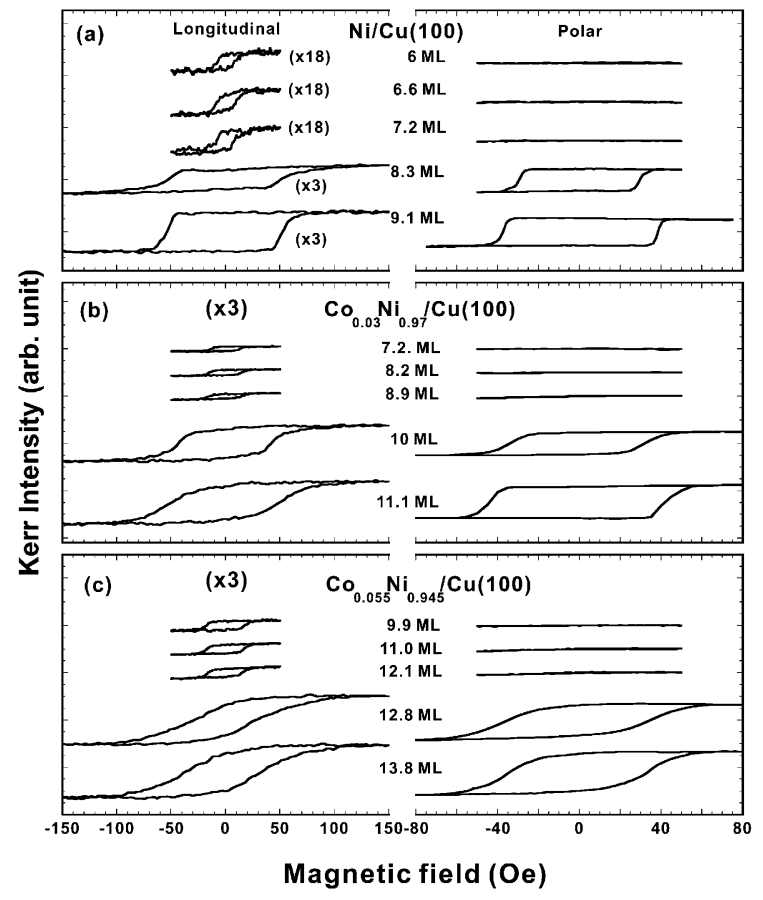

Fig. 7. Magnetic Kerr hysteresis loops taken at $110 \mathrm{~K}$ for (a) $\mathrm{Ni} / \mathrm{Cu}(100)$ (b) $\mathrm{Co}_{0.03} \mathrm{Ni}_{0.97} / \mathrm{Cu}(100)$ and (c) $\mathrm{Co}_{0.055} \mathrm{Ni}_{0.945} /$ $\mathrm{Cu}(100)$. The SRT with the increasing thickness can be observed clearly, and the $d_{\mathrm{c}}$ are about 7.5, 9.5 and 12.5 ML for these cases respectively. It should be noted that the unit of polar remanence was three times that of longitudinal one because the polar effect had much larger sensitivity.

mens. Above the $d_{\mathrm{c}}$, every series of magnetic films presented a dramatic increase both in remanence and coercive field in the longitudinal component as compared to those below $d_{\mathrm{c}}$. Meanwhile the magnetization in the polar component also began to appear (Fig. 7). Similar phenomenon was also found in earlier MOKE measurement of $\mathrm{Ni}$ / $\mathrm{Cu}(100)[5,16,26]$, but it is not realized very well so far. In general, the presence of both components of magnetization could be due to a canted easy axis state, or a distribution of in-plane and out-of-plane multidomain. It was thought to be the case of multidomain in previous discussion because of the $100 \%$ polar remanence. However, this conclusion lacks direct evidences.

In this experiment, when performing the MOKE measurement, the magnetic field was controlled slowly from positive maximum magnetic field to 
zero in the last step to ensure its final state is aligned in the upward direction with respect to the plane. Therefore the final state of the moment was aligned along the positive field direction, which means that if the MOKE measurement in this geometry is repeated again, its initial state should also be aligned along the positive magnetic field. However, when the geometry was switched from polar (in-plane) to in-plane (polar), then back to polar (in-plane) component, it was observed that while measuring the in-plane (polar) component, the magnetization of polar (in-plane) was changed simultaneously from fully positive saturation to fully negative saturation. It has been checked that the above situation does not result from the component of magnetic field since both the coercive field of the two directions are less than 50 Oe. Moreover, if it were multidomain, the moments in the two components should be independent and would not be influenced by magnetic field in the other direction and thus only a canted state can achieve the situation mentioned above. Therefore the coexistence of polar and longitudinal components is believed to be due to a canted state when the thickness is larger than $d_{\mathrm{c}}$. Besides, the abrupt increase in the in-plane coercive field is due to the reorientation transition of the easy axis from the film plane to canted direction. Also because of the canted moment, the polar Kerr effect has contribution in the hysteresis loops when measuring the in-plane MOKE. The sensitivity of the polar Kerr effect is several times that of the longitudinal Kerr effect [26]. Therefore an abrupt increase in the remanence of the in-plane magnetization is observed. Recently several models [2729] recommended that if the second order term anisotropy is taken into account, canted magnetization may appear. This could be also the origin of the canted state found here.

Correspondingly, the magnetic remanence of both perpendicular and in-plane components for various alloy films measured at $110 \mathrm{~K}$ were summarized in Fig. 8. The critical thickness of SRT in alloy ultrathin films $\mathrm{Co}_{x} \mathrm{Ni}_{1-x} / \mathrm{Cu}(100)$ were about 7.5, 9.5, 12.5 and $16.5 \mathrm{ML}$ for $x=0 \%, 3 \%, 5.5 \%$, and $8 \%$, respectively. For $x=10 \%$, alloy films always revealed in-plane anisotropy at least up to 20.6 ML. Additionally, the change of $d_{\mathrm{c}}$ with the

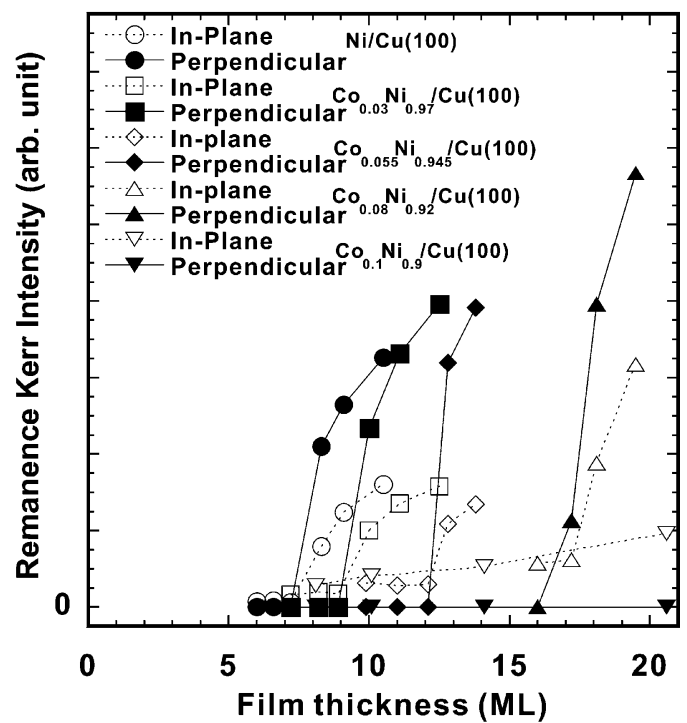

Fig. 8. Perpendicular and in-plane remanence Kerr effect of various alloy films $\mathrm{Co}_{x} \mathrm{Ni}_{1-x} / \mathrm{Cu}(100)$ measured at $110 \mathrm{~K}$. It can be clear observed that the critical thickness $d_{\mathrm{c}}$ was significantly delayed by increasing only a few Co concentration. Above $d_{\mathrm{c}}$, both the perpendicular and in-plane remanence had a dramatic increase. It should be noted that the unit of polar remanence was three times that of longitudinal one.

variation of measurement temperature from 110 to $350 \mathrm{~K}$ was within $1 \mathrm{ML}$. This result was compatible with prior work for the $\mathrm{Ni} / \mathrm{Cu}\left(\begin{array}{lll}1 & 0 & 0\end{array}\right)$ films [30] and will be discussed in our forthcoming publication [31].

The MEED and LEED data of the alloy films are analyzed to be of the same feature and although there exists SRT, their vertical interlayer distances are always of the same value within the error bars. We can thus conclude that SRT has nothing to do with the change in the crystalline structure and this conclusion is consistent with previous study of $\mathrm{Ni} / \mathrm{Cu}\left(\begin{array}{lll}1 & 0 & 0\end{array}\right)$ [21,22]. Besides, another experiment also indicated that even the rough film which was deposited at low temperature can only delay the $d_{\mathrm{c}}$ about 1 ML [21], so we can assume that the effect of roughness on $d_{\mathrm{c}}$ should be negligible in our system. Furthermore, since the mismatch of $\mathrm{Co}$ and $\mathrm{Ni}$ on $\mathrm{Cu}(100)$ are $-1.9 \%$ and $-2.5 \%$ respectively, in such little difference of Co concentration $(\leqslant 10 \%)$, the change of the strain $(0.06 \%)$ is not the dominating origin 
of the composition-induced effect and is should be negligible. Therefore what influences the straininduced anisotropy most should be the intrinsic change of magnetoelastic coefficient. Recently, a theoretical calculation of the magnetoelastic anisotropy in $3 \mathrm{~d}$ binary alloy has indicated the dramatic changes from the perpendicular magnetization to in-plane with only a few percent of Co concentration [12]. On the other hand, the change of magnetocrystalline anisotropy in such little variation of alloy composition is negligible as compared with the change of magnetoelastic anisotropy [14,33]. The later is about 10 times the former. Because the magnetic moment of $\mathrm{Co}-\mathrm{Ni}$ alloy changes linearly from the value of $\mathrm{Ni} 0.6 \mu_{\mathrm{B}}$ to that of Co $1.8 \mu_{\mathrm{B}}$ [32], the demagnetization energy has no dramatic change for the alloy films with such low Co concentration. So the straininduced magnetoelastic anisotropy which abruptly changes sign in the range of very low Co concentration should be responsible for the significant delay of the $d_{\mathrm{c}}$ of SRT in alloy films.

In previous reports $[22,34]$, the $\mathrm{CO}$ contamination might relax the structure of the top layer and reduce the magnetization and Curie temperature. The CO contamination had stronger effect for thinner films and it reduced the magnetic moment more when the temperature got closer to Curie point. However, for the $\mathrm{Co}-\mathrm{Ni}$ alloy films in this experiment, almost their thickness were more than $10 \mathrm{ML}$ and the MOKE measurement was performed at $110 \mathrm{~K}$ which was nearly in the region of saturated magnetization and far from Curie temperature. Thus the possible effect of CO contamination was reduced to the least and not enough to influence the conclusion. In another aspect, the possible relaxation induced by $\mathrm{CO}$ in the top layer may only result in the change of the surface term anisotropy and the volume term will be the same. The possible change of the surface anisotropy was not strong enough to result in the significant delay of SRT for Co-Ni alloy films. Therefore the conclusion about the abrupt change of the volume anisotropy with $3 \mathrm{~d}$ electron number was still supposed to be right. As the AES simulation was concerned, the little CO signal must have been added into the simulation through the reference data of various $\mathrm{ML} \mathrm{Co} / \mathrm{Cu}\left(\begin{array}{lll}1 & 0 & 0\end{array}\right)$ and $\mathrm{Ni} / \mathrm{Cu}\left(\begin{array}{lll}1 & 0 & 0\end{array}\right)$.
Finally, this simulation can still match the experimental data of alloy films.

\section{Summary}

Alloy ultrathin films $\mathrm{Co}_{x} \mathrm{Ni}_{1-x} / \mathrm{Cu}(100)$ with $0 \% \leqslant x \leqslant 10 \%$ up to $20.6 \mathrm{ML}$ were prepared in precise thickness and alloy composition. The growth mode of alloy films was characterized as the layer-by-layer growth until 4th monolayer, which was dominated by the feature of pure Ni films. All the films had the same features in LEED patterns denoting the apparent square islands with edges aligned to [ $\left[\begin{array}{ll}0 & 1\end{array}\right]$ and $\left[\begin{array}{lll}0 & 1 & 1\end{array}\right]$, consistent with the previous result of $\mathrm{Ni} / \mathrm{Cu}\left(\begin{array}{lll}1 & 0 & 0\end{array}\right)$ monitored by STM. The quantitative $I / V$-LEED analysis showed no significant difference in crystalline structure in the alloy films. Conclusively, in this series of alloy ultrathin films $\mathrm{Co}_{x} \mathrm{Ni}_{1-x} / \mathrm{Cu}(100), 0 \% \leqslant x \leqslant 10 \%$, the growth mode, morphology and crystalline structure do not change with the alloy composition.

The critical thickness $d_{\mathrm{c}}$ of SRT was strongly delayed by only few percent of Co concentration. For the Co concentration $x=0 \%, 3 \%, 5.5 \%$ and $8 \%$, the critical thickness of SRT were about 7.5 , 9.5, 12.5 and 16.5 ML, respectively. For $x=10 \%$, alloy films showed only the in-plane anisotropy with increasing thickness, the same as found in $\mathrm{Co} /$ $\mathrm{Cu}(100)$. This extremely sensitive SRT behavior is believed to result from the change of magnetoelastic anisotropy induced by tuning the average $3 \mathrm{~d}$ electron number at variation of Co composition.

Finally, the coexistence of the polar and longitudinal components were shown to be due to the presence of canted moment rather than magnetic microstructure of multidomain.

\section{Acknowledgements}

This work was supported by the National Science Council of Taiwan under grant no. NSC 892112-M-002-019 and by the Ministry of Education. 


\section{References}

[1] S. Müller, P. Bayer, C. Reischl, K. Heiz, B. Feldmann, H. Zillgen, M. Wuttig, Phys. Rev. Lett 74 (1995) 765.

[2] J. Thiele, C. Boeglin, K. Hricovini, F. Chevrier, Phys. Rev. B 53 (1996) R11934.

[3] R. Allenspach, M. Stampanoni, A. Bischof, Phys. Rev. Lett 65 (1990) 3344.

[4] B. Schulz, K. Baberschke, Phys. Rev. B 50 (13) (1994) 467.

[5] G. Bochi, C.A. Ballentine, H.E. Inglefield, C.V. Thompson, R.C. O'Handley, Phys. Rev. B 52 (1995) 7311.

[6] R. Jungbult, M.T. Johnson, J. aan de Stegge, A. Reinders, C. Chappert, J. Appl. Phys. 75 (1994) 6424.

[7] W.L. O'Brien, T. Droubay, B.P. Tonner, Phys. Rev. B 54 (1996) 9297.

[8] C.M. Schneider, P. Bressler, P. Schuster, J. Kirschner, J.J. de Migue, R. Miranda, Phys. Rev. Lett. 64 (1990) 1059.

[9] M. Kowalewski, C.M. Schneider, B. Heinrich, Phys. Rev. B 47 (1993) 8748.

[10] A.B. Shick, D.L. Novikov, A.J. Freeman, J. Appl. Phys. 83 (1998) 7258.

[11] H. Fujiwara, H. Kadomatsu, T. Tokunaga, J. Magn. Magn. Mater. 31-34 (1983) 809.

[12] P. James, O. Eriksson, O. Hjaortstam, B. Johansson, L. Nordström, Appl. Phys. Lett. 76 (2000) 915.

[13] P. James, O. Eriksson, B. Johansson, I.A. Abrikosov, Phys. Rev. B 59 (1999) 419.

[14] A. Lessard, T.H. Moos, W. Hüber, Phys. Rev. B 56 (1997) 2594.

[15] A. Dittschar, M. Kuch, M.-T. Lin, C.M. Schneider, J. Kirschner, Phys. Rev. B 57 (1998) R3209.

[16] F. Huang, M.T. Kief, G.J. Mankey, R.F. Willis, Phys. Rev. B 49 (1994) 3962.

[17] C.C. Chang, Surf. Sci. 48 (1975) 9.
[18] M.-T. Lin, J. Shen, W. Kuch, H. Jenniches, M. Klaua, C.M. Schneider, J. Kirschner, Surf. Sci. 410 (1998) 290.

[19] M. Zharnikov, A. Dittschar, W. Kuch, C.M. Schneider, J. Kirschner, Phys. Rev. Lett. 76 (1996) 4620.

[20] M.-T. Lin, J. Shen, W. Kuch, H. Jenniches, M. Klaua, C.M. Schneider, J. Kirschner, Phys. Rev. B 55 (1997) 5886.

[21] M. Zheng, J. Shen, P. Ohresser, Ch.V. Mohan, M. Klaua, J. Barthel, J. Kirschner, J. Appl. Phys. 85 (1999) 5060.

[22] W. Platow, U. Bovensiepen, P. Poulopoulos, M. Farle, K. Babschke, Phys. Rev. B 59 (12) (1999) 641.

[23] J. Shen, J. Giergiel, J. Kirschner, Phys. Rev. B 52 (1995) 8454.

[24] J. Shen, M.-T. Lin, J. Giergiel, C. Schmidthals, M. Zharnikov, C.M. Schneider, J. Kirschner, J. Magn. Magn. Mater. 156 (1996) 104.

[25] J.W. Matthews, J.L. Crawford, Thin Solid Films 5 (1970) 187.

[26] S.Z. Wu, G.J. Mankey, F. Huang, R.F. Willis, J. Appl. Phys. 76 (1994) 6434.

[27] M. Farle, B. Mirwald-Schulz, A.N. Anisimov, W. Platow, K. Baberschke, Phys. Rev. B 55 (1997) 3708.

[28] Y.T. Millev, H.P. Oepen, J. Kirschner, Phys. Rev. B 57 (1998) 5848.

[29] K. Ha, R.C. O’Handley, J. Appl. Phys. 87 (2000) 5944.

[30] M. Farle, W. Platow, A.N. Anisimov, P. Poulopoulos, K. Baberschke, Phys. Rev. B 56 (1997) 5100.

[31] C.C. Kuo, W.C. Lin, C.L. Chiu, Minn-Tsong Lin, in press.

[32] C. Kittel, in: Introduction to Solid State Physics, Seventh edition, Wiley, New York, 1996, p. 457.

[33] G.H.O. Daalderop, P.J. Kelly, M.F.H. Schuurmans, Phys. Rev. B 41 (11) (1990) 919.

[34] F. Huang, G.J. Mankey, R.F. Willis, Surf. Sci. Lett. 297 (1993) L79. 